\title{
CULTURAS COSMOPOLITAS DEL PARTO. CONTRASTANDO SUS BASES ANTROPOLÓGICAS DESDE LA PERSPECTIVA BIOÉTICA
}

\author{
José Manuel Hernández Garre¹, Baldomero de Maya Sánchez ${ }^{1}$
}

Resumen: El objetivo del presente artículo ha sido explorar, desde la perspectiva ética, las principales características y bases antropológicas del parto, tratando de dilucidar, desde un posicionamiento personalista, qué patrones son más consecuentes con el respeto de la dignidad materna y los principios básicos de la bioética. La metodología elegida fue el análisis documental, mediante el uso de diferentes bases de datos del ámbito de las ciencias médicas, sociales y antropológicas. Los resultados muestran abordajes bioéticamente antagónicos, situándose, de una parte, dos reduccionismos ontológicos de corte biologicista: el parto medicalizado y el parto natural, y, de otra, un abordaje holístico: el parto humanizado. Los dos primeros rompen con el principio de autonomía y no maleficiencia, al quedar el destino de las madres marcado por su fisiología, naturaleza que, desde la perspectiva medicalizada, es representada desde las nociones de "riesgo" y "fragilidad", quedando las madres expuestas a múltiples intervenciones clínicas iatrogénicas; mientras que, desde la cosmovisión naturista, es percibida desde la noción de "infalibilidad", lo que las convierte en rehenes de su supuesta perfección anatómica. Conflictos éticos que no surgen en el modelo humanista, al representar éste a las madres como sujetos sociales globales, que exceden cualquier reduccionismo atomista.

Palabras clave: culturas del parto, parto medicalizado, parto natural, parto humanizado, personalismo

\section{Cosmopolitan cultures of childbirth. Contrasting its anthropological bases from the bioethical perspective}

\begin{abstract}
The objective of this article has been to explore, from an ethical perspective, the main anthropological characteristics and bases of childbirth, trying to elucidate, from a personalist position, which patterns are more consistent with respect for maternal dignity and basic principles of bioethics. The methodology chosen was the documentary analysis, through the use of different databases in the field of medical, social and anthropological sciences. The results show bioethically antagonistic approaches, placing, on the one hand, two ontological reductions of a biological nature: medicalized and natural birth, and, on the other, a holistic approach: humanized birth. The first two break with the principle of autonomy and non-maleficiency, as the fate of the mothers is marked by their physiology, a nature that, from a medicalized perspective, is represented from the notions of "risk" and "fragility", leaving the mothers exposed to multiple iatrogenic clinical interventions; while, from the naturist worldview, it is perceived from the notion of "infallibility", which makes them hostages of their supposed anatomical perfection. Ethical conflicts that do not arise in the humanist model, when it represents mothers as global social subjects, who exceed any atomistic reductionism.
\end{abstract}

Keywords: birth cultures, medicalized delivery, natural birth, humanized childbirth, personalism

\section{Culturas cosmopolitas do parto. Contrastando suas bases antropológicas desde uma perspectiva bioética}

Resumo: O objetivo do presente artigo foi explorar, desde uma perspectiva ética, as principais características e bases antropológicas do parto, tratando de elucidar, desde um posicionamento personalista, que padróes são mais consistentes com o respeito da dignidade materna e os princípios básicos da bioética. A metodologia eleita foi a análise documental, mediante o uso de diferentes bases de dados do âmbito das ciências médicas, sociais e antropológicas. Os resultados mostram abordagens bioeticamente antagônicas, situando-se de um lado dois reducionismos ontológicos de corte biologicista, o parto medicalizado e natural, e de outro uma abordagem holística, o parto humanizado. Os dois primeiros rompem com o princípioi da autonomia e não maleficência, ao deixar o destino das mães marcado por sua fisiologia, natureza que, desde uma perspectiva medicalizada, está representada pela noção de risco e fragilidade, ficando as mães expostas a múltiplas intervenções clínicas iatrogênicas; enquanto que, desde uma cosmovisão naturista, é percebida desde a noção de infalibilidade, o que converte as mães em reféns de sua suposta perfeição anatômica. Conflitos éticos que não surgem no modelo humanista, ao representar este às mães como um sujeito social global que excede qualquer reducionismo atomista.

Palavras chave: culturas do parto, parto medicalizado, parto natural, parto humanizado, personalism

\footnotetext{
${ }^{1}$ Departamento de Antropología Social, Universidad de Murcia, España

Correspondencia: josemanuelhernandezgarre@gmail.com
} 


\section{Introducción}

Los individuos "pueden participar de muchas y variadas culturas y sustentar distintas formas de identidad"(1:109). Identidades que se manifiestan en procesos como el nacimiento, el cual es pensado y representado de diferentes maneras, al tratarse de un hecho cultural y no solo biológico, en el que se pone en evidencia la ambigüedad de la interacción entre estas dos dimensiones(2). Las cosmovisiones del parto no se producen, pues, al azar, sino que se forman en un marco biosocial, conformado tanto por factores biológicos universales como por factores culturales(3). Determinantes que se agrupan en auténticas "etno obstetricias"(4) o "culturas del parto", que recogen toda la serie de "conocimientos, costumbres, creencias, valores, prácticas y experiencias que, (...) los actores [del parto] manifiestan (...) respecto al nacimiento"(5:10). Estamos ante las diferentes maneras en que cada cultura particular concibe un hecho biológico como es el nacimiento, ante un fenómeno de estudio catalogado bajo el epígrafe de "antropología del nacimiento», cuyo objetivo es testimoniar las diferentes representaciones y prácticas culturales que vertebran las distintas sociedades para entenderlo. Se trata, en definitiva, de todo un corpus de conocimiento, cuyo inicio sitúan algunos autores(6) en los trabajos de la antropóloga americana Margaret Mead y que ha dado lugar a una prolífica literatura científica en las últimas décadas. Estudios como los de Jordan(3), Pizzini(7), Knibiehler y Fouquet(8), Donnison(9), Ortiz(10,11), Narotzky(12), Esteban(13), Bodoque(14), Montes(5), Imaz(15), Blázquez(16) o Hernández(17), centrados en el análisis antropológico de los procesos reproductivos.

Como vemos, no se trata de una materia nueva, sino de un fenómeno estudiado dentro de la antropología desde diferentes perspectivas, y que ha permitido caracterizar los diferentes paradigmas y conjuntos de prácticas autorizadas para dar cobertura a los mismos. Cosmovisiones que se traducen en verdaderos modelos culturales de asistencia a los partos, conformando diferentes "culturas del nacimiento" o etno obstetrcias, como el parto hospitalario e intervencionista, consecuente con un paradigma tecnocrático medicalizado; el parto no institucionalizado o domiciliario, propio de una cosmovisión naturista, o el parto hospitalario humanizado, consecuente con una perspectiva holístico-humanista(18:16).

En este marco de pugna ideológica entre culturas cosmopolitas del parto, surge el interés del presente artículo, cuyo objetivo ha sido comparar, desde la perspectiva bioética, sus características definitorias y bases antropológicas, tratando de dilucidar qué patrones son más consecuentes con el respecto a la dignidad del binomio materno-fetal y a los principios bioéticos básicos. Los análisis se han realizado desde una perspectiva crítica posmoderna que, siguiendo las premisas de la antropología personalista, trata de poner de relieve la defensa de la persona como centro y receptáculo de dignidad inalienable.

\section{Metodología}

Se trata de un estudio descriptivo, de carácter cualitativo, basado en la realización de un análisis documental. La información se recabó durante 2018, mediante el examen de diversos documentos de ciencias médicas, sociológicas y antropológicas. Las bases de datos consultadas fueron Pudmed, CINAHl, Cochrane, Antro Base: Social and Cultural Anthropology, Current Contens, SOCAntropología social y Periodicals Archive Online - PAO_. Durante las búsquedas, se consultaron los tesaurus de las diferentes bases de datos y, en su defecto, el Medical Subject Headings (MeSH), seleccionándose como descriptores los siguientes términos: birth cultures, humanized childbirth, natural birth y medicalized delivery. Respecto de la estrategia de las búsquedas, se seleccionaron únicamente aquellos artículos en inglés o español en los cuales los descriptores aparecían en el título o el abstract, no se estableció restricción en razón de fecha, con el objetivo de recabar la mayor información posible. Por último, en cuanto al análisis de contenido, se seleccionaron aquellos artículos de revistas de producción científica relacionados con la temática de estudio, consultándose también otros documentos de interés, como libros, estrategias de atención, guías de práctica clínica e información perteneciente a circuitos informales vertida por asociaciones relacionadas con la maternidad a través de folletos o páginas web. 


\section{Resultados}

Del análisis documental devienen dos categorías de análisis que vertebran la bibliografía sobre el tema, de una parte, las características definitorias que enmarcan estas tres culturas del parto contemporáneas y, de otra, las bases filosófico-antropológicas en las que se sustenta su difusión y representación social colectiva.

\section{Características definitorias}

Los atributos que caracterizan cada una de estas etno obstetricias difieren, en el sentido de que representan formas heterogéneas, cuando no antagónicas o contrapuestas, de representar tanto el nacimiento en sí mismo como el entorno, los objetivos clínicos y la ideación del estatus maternofetal a lo largo del proceso.

\section{El parto medicalizado o intervencionista}

El nudo gordiano que atraviesa esta cultura del nacimiento es un biologicismo en clave negativa. La biología es percibida como frágil e imperfecta, por lo que hay que intervenir desaforadamente para minimizar riesgos. Se trata de un modelo cultural de asistencia, caracterizado por atributos como la medicalización, la institucionalización, la fragmentación del cuidado, la tecnologización o el monopolio médico $(19,20)$. Sus orígenes se sitúan en el proceso de hospitalización del parto que se produjo a partir de la segunda mitad del siglo $\mathrm{XX}(21,22)$, lugar privilegiado para la medicalización colectiva $(23,24)$ y la vigilancia en aras de la disminución de riesgo y la supresión del dolor(5). Los nacimientos son concebidos, pues, como procesos científicos altamente tecnológicos, que cada vez se distancian más de los conocimientos y estrategias de afrontamiento de las mujeres(25).

Estamos ante el patrón de parto mayoritario y legitimado en las sociedades industriales(26), seguramente de la mano de la supremacía que actualmente ostenta el modelo biomédico(27), pese a que —está demostrado- su uso indiscriminado incrementa la morbimortalidad maternofetal(28-31). Se trata, en definitiva, de una etno obstetricia llevada a cabo bajo un monopolio biomédico, que ha empleado su poder para que las mujeres acepten sus decisiones al poseer los "conocimientos autorizados" (32:319). Lo importante es aportar datos objetivos sobre las desviaciones biológicas del parto, corolario de una lógica productivista que tiende a la "uniformización" del proceso (33), que busca la condición de asistabilidad. Estatus que "no depende necesariamente de un substrato biológico o psíquico, sino de la existencia de un conjunto de reglas, necesariamente arbitrarias" (34:140), cuyo objetivo final es garantizar una asistencia institucional y uniforme.

\section{El parto natural}

Esta cosmovisión surge de dos dimensiones narratológicas dispares, la sacralización de la biología materna y el empoderamiento femenino. Al contrario que en el caso anterior, el binomio materno-fetal es concebido como un engranaje biológico perfecto, que se complementa de forma natural durante el parto sin la necesidad de intervenciones externas. En este sentido, toda intromisión biotecnológica es concebida como un desencadenante iatrogénico que puede dificultar el proceso, como una interferencia en el vínculo materno-fetal. Se parte de posiciones primatológicas que ensalzan la dimensión reguladora natural de la gestación materna, ligando las intromisiones obstétricas a ideologías biomédicas patriarcales, cuya función va más allá del control de riesgos, para situarse en la colonización tecnológica de la reproducción y el organismo femenino. Desde estos postulados, se demanda un empoderamiento de la mujer que le lleve a reconquistar su territorio corporal, a afrontar sus partos desde la seguridad en sí misma, recuperando así el protagonismo perdido. La autoridad sobre temas de reproducción pertenece, pues, a las madres, y ciencia y tecnología tienen que estar al servicio de éstas(35-41).

En esta línea, intervenciones como la administración exógena de oxitocina, la rotura artificial de membranas, la instrumentación de los partos o la administración de analgesia epidural son criticadas, al considerar que alteran los ritmos endocrinos, hormonales y biomecánicas naturales del parto. El proceder adecuado sería, pues, el de una conducta expectante, que ceda totalmente el protagonismo a las madres, madres que no solo se convierten en sujeto activo de sus partos, sino 
en el elemento esencial que dirige el proceso mediante la escucha de sus sensaciones y del recurso a sus instintos primigenios. El lema final es la empoderación de la propia corporalidad femenina y el hads-off asistencial, realidades que solo se pueden conseguir desde esta perspectiva mediante una desinstitucionalización de los partos, que los retorne al lugar del que nunca debieron salir: el hogar de las madres.

\section{El parto humanizado}

Por último, el patrón humanizado apuesta por una superación de la visión del parto exclusivamente biologicista, abriéndose a perspectivas más holísticas que integren también las dimensiones psicológicas y socioculturales de las parturientas. El objetivo no es pues desinstitucionalizar los partos de los hospitales, como en el caso anterior, sino conseguir un equilibrio entre las necesidades de las madres y las de los profesionales, buscando un modelo en el que la tecnología se complemente con el humanismo asistencial. Se parte de una defensa ontológica de la dignidad inalienable de las madres, de una unidad mente, cuerpo y espíritu que las reintegra en su totalidad y en el contexto en el que se produce el proceso. Desde esta atalaya, cobran especial importancia aspectos como la promoción de un entorno adecuado, libre de ruidos y luces molestas, la autonomía, el respeto a la intimidad, el acompañamiento de los familiares, la libertad de movimientos, la utilización de pelotas de goma o de bañeras o el abordaje del dolor desde estrategias multidimensionales que no se limiten al uso de la analgesia epidural(42). En definitiva, se considera cada parto como un proceso único, que ha de abordarse desde la individualización asistencial, desde la comprensión de las disimiles variables biopsicosociales que acontecen en cada nacimiento y desde la comprensión de que las mujeres son un sujeto activo del parto(18).

Estamos ante una cultura del nacimiento, surgida como reacción a la tendencia biomédica de intervenir excesivamente en los partos de bajo riesgo en los hospitales. Ante una cruzada encabezada por la propia Organización Mundial de la Salud (OMS), que en diferentes documentos $(43,44)$ ha apostado por una mayor humanización de los partos hospitalarios, que evite la tasa de cesáreas e inducciones de parto injustificadas y promocione la consecución de partos normales, a la vez que insiste en la promoción de investigaciones que evalúen las praxis y tecnologías de atención al parto más adecuadas. Recomendaciones que han sido secundadas en España por diferentes organismos, como el Ministerio de Sanidad y Consumo(45-47), la Sociedad Española de Ginecología y Obstetricia(48) o la Federación de Asociaciones de Matronas de España(49), que insisten en la necesidad de evitar la medicalización excesiva de los partos normales, situando su excelencia en aspectos como el control de riesgos, la actitud expectante, la promoción de un entorno humanizado y el respeto a las necesidades emocionales de las madres.

\section{Bases antropológicas}

Los atributos de cada una de estas etno obstetricias no es más que la punta del iceberg de toda una serie de filosofías, perspectivas etnohistóricas y postulados antropológicos que las fundamentan ideológicamente.

\section{Etno obstetricias tencocráticas}

El abordaje tecnocrático del parto parte de filosofías materialistas, como el atomismo de Demócrito y Leucipo, para encontrar su piedra angular en la escisión entre res extensa y res cogitans, en ese dualismo cartesiano que tiende a concebir el cuerpo como una posesión del alma. Se trata de perspectivas biologicistas, de reduccionismos ontológicos que apuntan a simplificar la inextricable complejidad del ser en cuanto a ente multidimensional e inmanente; de proyectos que encuentran su estatus de posibilidad con el nacimiento, a finales del siglo XIX, de una medicina experimental desligada de sus componentes subjetivos y centrada en los hechos empíricos constatables de la corporalidad. Desde estos posicionamientos se piensa que el ser humano no trasciende lo biológico, que el cuerpo-sujeto no excede todas las posibilidades de investigación(50,51).

Perspectivas materialistas que han sido llevadas al extremo por ciertos postulados posthumanistas y transhumanistas, que pretenden curar al cuerpo de sus debilidades constitutivas a través del uso indiscriminado de la tecnología(52). Estamos ante posiciones antitéticas a los mecanicismos del 
siglo XVIII que reverenciaban el cuerpo; ahora se identifica la naturaleza humana con la vulnerabilidad, por lo que se la rechaza(53), pretendiendo hacer tanteos de mejora con ella(54). Se proclama, en definitiva, el ocaso de la biología humana, desde una clara exaltación baconiana que se declara protecnológica y antibiologicista(55-57). Y todo ello sazonado, a su vez, por posicionamientos biopolíticos, que aspiran a un control estatal de la reproducción, desde el que la ciencia se imponga al individuo, sano o enfermo, en un acto de autoridad(23). Se trata de una domesticación de los cuerpos gestantes según las necesidades del orden social y político(58); de una hegemonía del poder técnico y profesional de los especialistas, mediante una serie de funciones de control y legitimación(59), que los inviste, en el nivel corporal, del mismo poder que el clero posee sobre las $\operatorname{almas}(60)$.

Por último, se esbozan también filosofías productivistas, se atisba la influencia de planteamientos tayloristas, desde los que la organización del trabajo obstétrico se basa en el cálculo y ajuste de los tiempos que permiten obtener el producto final al mejor coste-beneficio. Desde esta perspectiva, la reproducción se convierte en un proceso más de producción, aunque su finalidad sea, en este caso, la producción de personas(16). El control férreo del producto, la mercantilización asistencial y la asignación de una serie de tiempos necesariamente arbitrarios, se convierten así en ejes centrales que atraviesan el proceso asistencial.

Se trata de toda una serie de disimiles perspectivas materialistas, biologicistas, posthumanistas, biopolíticas y tayloristas, desde las que se construyen las características principales de este patrón, es decir, la medicalización, tecnologización, burocratización y mecanización del parto.

\section{Etno obstetricias naturistas}

El naturismo obstétrico encuentra su primordio en las nociones hipocráticas de una terapéutica basada en el respeto de las fuerzas naturales, en la concepción de las propias defensas corporales como fuente de curación de las enfermedades. Se trata de una clara apuesta por la conducta expectante, por poner a la persona en las mejores condiciones para que la naturaleza la sane, partiendo de la premisa de que lo primero es no hacer daño. Postura que también encontramos en la terapeútica de Paracelso o en el modelo del entorno de Florence Nigthingale y su apuesta por la sanación mediante el control de los elementos contextuales(61). Estamos ante la vuelta al mito del buen salvaje de Rousseau, ante una vuelta a los orígenes naturales del hombre lejos de la contaminación de la sociedad tecnocrática(62). Se trata de una reverenciación de la naturaleza corporal, similar a la que postulaban mecanicistas del siglo XVII, como La Mettrie, Helvetius o D'Holbach, perspectiva desde la que el cuerpo se concibe como una máquina corporal perfectamente coordinada que no precisa de la intervención externa.

Otra de las corrientes de pensamiento que vertebra esta etno obstetricia es la corriente ecofeminista, movimiento partícipe de una tercera ola de feminismo, que defiende la existencia de múltiples modelos de mujer determinados por cuestiones sociales, étnicas, de clase social, de orientación sexual o de religión(63). El ecofeminismo nace como reacción a lo que las feministas definen como una apropiacion masculina de la reproducción, reflejo del patriarcado occidental(64). En esta línea, el uso indiscriminado de la biotecnología en los partos es concebido como expresión de un modelo médico masculino, que no tiene en cuenta los recursos e instintos reproductivos.

Estamos pues ante un patrón cultural del nacimiento sustentado en dos pilares: la mitificación del binomio materno-fetal y la concepción del intervencionismo biomédico como expresión patriarcal de apropiación de la reproducción. Enfoque defendido por autores como Odent(35-37) o Kitzinger (38-41), que apuestan por una vuelta a los instintos primatológicos impresos de forma natural en la maternidad, por un patrón asistencial del parto domiciliario que respete los ritmos innatos del lance.

\section{Etno obstetricias holístico-humanistas}

La concepción holística del parto nace de una visión global de la persona, de una representación aristotélica de alma y cuerpo como parte de una misma sustancia, como un compuesto indisoluble de materia y forma. En este sentido, el ser trasciende la corporalidad en la medida en que ésta y 
sus dimensiones insustanciales forman una unión inmanente. Perspectivas humanistas influidas también por las concepciones judeocristianas del cuidado como actividad caritativa, o por las corrientes filosóficas encarnadas en el personalismo.

Estamos ante una visión del hombre como un ser relacional, esencialmente social y comunitario, como un ser libre, trascendente y con un valor en sí mismo que le impide convertirse en medio para los fines de otros(65). Ello supone un respeto a su dignidad inalienable, una posición que huye de cualquier mitificación de la técnica que deshumanice los procesos asistenciales. Que se opone, en definitiva, al intento de reducir la corporalidad a un simple conjunto de funciones fisiológicas o biomecánicas(66). Atalaya desde la cual la persona es un ser moral, capaz de amar, de actuar en función de una actualización de sus potencias y, finalmente, de definirse a sí mismo considerando siempre la naturaleza que le determina. Ello implica una huida de cualquier cosificación clínica que le instrumentalice, que socave su dignidad y autonomía(67).

A la sazón de estos posicionamientos, se unen también los postulados fenomenológicos husserlianos, que ponen el énfasis en la diferencia entre los que las cosas son y cómo se hacen conscientes en nuestra mente, abriendo el camino a la subjetividad humana, a la individualización asistencial según las diferentes perspectivas maternas. $\mathrm{O}$ los conceptos existencialistas kierkegaardianos, que sitúan a la persona como un "ser en el mundo", cuya existencia real se materializa a través de la libertad personal, lo que pone el dedo en la responsabilidad individual, en el derecho de autonomía de las pacientes. Por último, cabe destacar los aportes hechos desde la escuela antropológica de cultura y personalidad, perspectivas boasianas que hacen hincapié en las disímiles cosmovisiones culturales. Premisa de la que parten las nociones de cuidado de Leininger, la concepción de que el cuidado es transcultural y, como tal, debe adaptarse según las preferencias culturales si quiere ser congruente(68).

Se dibujan así las filosofías etnohistóricas que perfilen las características cardinales de esta etno obstetricia: el holismo asistencial, la defensa ontológica de la dignidad del binomio materno-fetal, el derecho a la individualización, la promoción de la autonomía y el respecto a las diferentes perspectivas culturales maternas. Se trata, en definitiva, de un modelo que va más allá de las clásicas nociones biomédicas centradas en la biología, para prolongar la asistencia hacia las dimensiones psicológicas y socioculturales del proceso, pero sin necesidad de sacar los partos del contexto hospitalario. Enfoque humanista que defienden en la actualidad autores como Jean Donnison(9), Emily Martin(26), Brigitte Jordan(3), Barbara Ehrenreich, Deirdre English(69) y Marsden Wagner(70).

\section{Discusión}

Del análisis de los resultados emanan tres patrones asistenciales del parto, tres etno obstetricias que dibujan heterogéneas perspectivas bioéticas de abordar un fenómeno biológico, pero a la vez cultural. En este sentido, germinan cosmovisiones antitéticas sustentadas en dispares perspectivas antropológicas, de las que, a su vez, subyacen diferentes consecuencias desde la mirada ética. De una parte, emergen dos biologicismos antagónicos: el tecnocrático, que identifica la naturaleza corporal con la vulnerabilidad, apostando por la intervención indiscriminada y la prevención de riesgos; y el naturismo, situado en una reverenciación de la biología materna, que sacraliza el cuerpo gestante y la actitud expectante. De otra parte, la perspectiva holística, que sitúa la excelencia en la humanización asistencial, la defensa de la dignidad materna y el abordaje biopsicosocial del proceso. Las limitaciones éticas de los dos primeros planteamientos encuentran su punto en común en la cosificación biológica que hacen de las madres en cuanto sujeto de actuación, en el sentido de que sus abordajes clínicos durante el parto quedan anclados en una noción atomista de la corporalidad. Queda así comprometido el principio de autonomía de las madres, al quedar su destino ligado a los procesos marcados por su fisiología, naturaleza que, desde la perspectiva medicalizada, es representada a partir de la noción de riesgo y fragilidad, quedando las madres expuestas a múltiples intervenciones clínicas, mientras que, desde la cosmovisión naturista, es percibida desde la noción de infalibilidad, lo que convierte a las madres en rehenes de su supuesta perfección anatómica. Se rompe así con el derecho de las madres de darse a sí mismas las reglas y normas durante el 
parto, al quedar cosificadas en un útero máquina que ha de dar a luz, o bien a través de toda una cascada de intervenciones impuestas por los clínicos — desde la perspectiva medicalizada - o sin ningún tipo de ayuda externa — desde la visión naturista- Por otro lado, también queda seriamente afectado el principio de no maleficiencia, basamen por el que cualquier intervención clínica debe partir de la noción básica de no generar efectos nocivos. En este sentido, tanto la excesiva intervención del modelo medicalizado, generadora de efectos iatrogénicos evitables(71), como la sacralización de la conducta expectante del modelo naturista, desde una supuesta e irrefutable perfección fisiológica, pueden generar daños a todas luces evitables. A ello se suma, en el caso del parto intervencionista, los riesgos derivados de las nociones productivistas, que se manejan sobre todo en los contextos hospitalarios privados, en los que se opta por procedimientos técnicamente complejos, como la cesárea, al ser mejor remunerados que los partos normales(72). Se rompe entonces con los principios de justicia, reconocimiento e inclusión social que merecen todas las madres, al dejar de considerarlas como principios en sí mismas, que no pueden ser objeto de intereses de ter$\operatorname{ceros}(65)$.

En oposición a estos planteamientos biologicistas se sitúa el abordaje humanista, posicionamiento que concibe a las madres como "seres" sociales y holísticos, que exceden las grafías propias de su dimensión corporal, quedando pues al margen de los riesgos bioéticos apuntados. Desde esta visión biopsicosocial no queda comprometido el principio de autonomía, al constituirse las madres en sujetos activos de su propio proceso; en este sentido, su destino no queda determinado por su vertiente fisiológica, ya sea desde las nociones de riesgo o infalibilidad, sino que depende de un proceso continuo de consenso entre las necesidades clínicas, emanadas del mantenimiento de unos estándares de seguridad aceptables, y sus propias necesidades. Se trata de un punto de equilibrio entre las demandas institucionales y las propias peticiones maternas, en el cual no se compromete su autonomía, sino que se intenta garantizar la seguridad del proceso sin sacar de la ecuación las preferencias y decisiones de las madres al respecto. Tampoco parece quedar comprometido el princi- pio de no maleficencia, ya que las decisiones no están necesariamente marcadas por posicionamientos ideológicos de exacerbación de la noción de riesgo o fragilidad materna, pero tampoco se sitúan en la sacralización de una biología materna que debiera de superar cualquier dificultad sin la intervención de la técnica. Lo que vertebra todo el tejido de esta representación es la consideración de la inalienable dignidad de las madres $(51,66,67)$, sin por ello caer en reduccionismos ontológicos que coarten el necesario control de riesgos de las praxis médicas contemporáneas.

Del análisis bioético de los tres patrones de parto cosmopolitas expuestos, surge la necesidad de articular itinerarios clínicos que se sustenten en el respecto de la dignidad materna, vías clínicas que no condenen a las mujeres a la intervención indiscriminada o a la sacralización de la conducta expectante, desde claros reduccionismos ideológicos biologicistas. Es el momento de vertebrar representaciones y praxis humanistas que, desde el respeto, garanticen la autonomía y no maleficencia en los procesos de atención obstétricos, sin por ello renunciar a un más que lógico control de riesgos. A ello ayudará esterilizar los procesos de atención de ciertas ideologías nocivas como la biopolítica, el posthumanismo, el productivismo, el ecofeminismo o el mecanicismo, en pro de fomentar marcos de representación personalista que respeten la dignidad de las madres sin cosificarlas desde visiones atomistas.

\section{Agradecimientos}

Al Departamento de Ciencias Políticas, Antropología Social y Demografía de la Universidad de Murcia, por el apoyo recibido durante la investigación. 
Culturas cosmopolitas del parto - José Manuel Hernández Garre, Baldomero de Maya Sánchez

\section{Referencias}

1. Comas D`Argemir D. Economía, cultura y cambio social. En Prat y Martínez (Eds.). Ensayos de Antropología Cultural. Barcelona: Ariel; 1996: 104-113.

2. Ranisio G. Venire al mondo. Credenze, pratiche, rituali del parto. Roma: Meltemi; 1998.

3. Jordan B. Birth in four cultures. A Crosscultural Investigation of Childbirth in Yucatan, Holland, Sweden and the United States. Montreal: Eden Press Women's Publications; 1993.

4. McClain C. Ethno-obstetrics in Ajijic. Anthropological Quarterly 1975; 48(1): 38-56.

5. Montes MJ. Las culturas del nacimiento. Representaciones y prácticas de las mujeres gestantes, comadronas y médicos. Tesis doctoral, Univesitat Rovira i Virgili, Tarragona; 2007.

6. Davis-Floyd R, Sargent C, (Eds.). Childbirth and authoritative knowledge. Cross-Cultural Perspectives. Los Ángeles: University of California Press; 1997.

7. Pizzini F. Il parto in ospedale: tragitto della donna e rituali dell'istituciones. En Pizzini (coord.), Sulla scena del parto: luoghi, figure, pratiche. Milano: Franco Angeli Editore; 1981: 129-147.

8. Knibiehler Y, Fouquet, C. Le femme et les médecins. Analyse Historique. París: Hachette; 1983.

9. Donnison J. Midwives and medical men. A History of Inter-professional rivalries and women's rights. London: Heinemann Educational Books, Ltd.; 1977.

10. Ortiz T. El discurso médico sobre las mujeres en la España del primer tercio del siglo veinte. En López Beltrán (coord.), Las mujeres en Andalucía. Málaga: Actas del $2^{\circ}$ encuentro interdisciplinar de estudios de la mujer en Andalucía. Vol. I; 1993: 109-138.

11. Ortiz T. Las matronas y la transmisión de saberes científicos sobre el parto en la España del siglo XIX. Arenal 1999; 6(1): 55-79.

12. Narotzky S. Mujer, mujeres, género. Una aproximación crítica al estudio de las mujeres en las ciencias sociales. Monografías, 14. Madrid: CSIC; 1995.

13. Esteban ML. Actitudes y percepciones de las mujeres respecto a su salud reproductiva y sexual. Necesidades de salud percibidas por las mujeres y respuesta del sistema sanitario. Tesis doctoral, Universidad de Barcelona, Barcelona; 1992.

14. Bodoque Y. Discursos y prácticas sobre sexualidad y reproducción. Tesis doctoral. Universidad Rovira i Virgili, Tarragona; 1996.

15. Imaz E. Mujeres Gestantes, madres en gestación. Representaciones, modelos y experiencias en el tránsito a la maternidad de las mujeres vascas contemporáneas. Tesis doctoral. Universidad del País Vasco, País Vasco; 2008.

16. Blázquez ML. Ideologías y prácticas de género en la atención sanitaria del embarazo, parto y puerperio: el caso del área 12 de la Comunidad de Madrid. Tesis Doctoral, Universidad d Rovira i Virgili, Tarragona; 2009.

17. Hernández JM. El parto hospitalario e intervencionista. Una construcción social de la maternidad. Tesis doctoral. Universidad de Murcia, Murcia; 2011.

18. Davis-Floyd R. The technocratic, humanistic, and holistic paradigms of child-birth. International Journalof Gynecology \& Obstetrics 2001; 75(1): 5-23.

19. Stoller N. Forced labor: maternity care in the United States. Oxford: Pergamon Studies in Critical Sociology; 1974.

20. Hernández JM, Echevarría P. La parte negada del parto institucionalizado. Explorando sus bases antropológicas. Revista de Dialectología y Tradiciones Populares 2014; 69(2): 327-348.

21. Vía R. Com neixen els catalans. Barcelona: Club editor El Pi de les tres Branques; 1972.

22. Hernández JM. Historia de las matronas. Evolución de la ciencia y el arte de la partería. Murcia: Diego Marín; 2012.

23. Foucault M. La vida de los hombres infames. Madrid: La Piqueta; 1990[1977].

24. Comelles JM. Fe, carisma y milagros. El poder de curar y la sacralización de la práctica médica contemporánea. En González Alcantud y Rodríguez Becerra (eds.). Creer y curar: la medicina popular. Granada: Biblioteca de Etnología Diputación Provincial de Granada; 1996: 301-336.

25. Oakley A. The captured Womb: a history of the Medical Care of Pregnant Women. Oxford: Basil Bernstein; 1984.

26. Martin E. The Woman in the Body. Boston: Beacon Press; 1987.

27. Menéndez EL. Antropología médica, orientaciones, desigualdades y transacciones. México: Cuadernos de la Casa Chata, $179 ; 1990$.

28. Olsen O. Meta-analysis of the safety of Home Birth. Birth. Issues in perinatal care 1997; 24(1): 4-13.

29. Campbell R y Macfarlane A. Where to be born? Oxford: Nacional Perinatology Epidemiology Unit; 1987.

30. Tew M. Place of birth and perinatal mortality. J R Coll Gen Pract 1985; 35: 390-394. 
31. Tew M. Safer childbirth: a Critical History of Maternity Care. Oxford: Oxford University Press; 1990.

32. Irwin S, Jordan B. Knowledge, Practice and power: court-ordered caesarean sections. Medical Anthropology Quarterly 1987; 1(3): 319-334.

33. Esteban ML. Reproducción del cuerpo femenino. Donostia: Tercera Prensa; 2001.

34. Comelles, JM. Sociedad, salud y enfermedad: los procesos asistenciales. Jano 1985; 655: 71-83.

35. Odent M. El bebé es un mamífero. Madrid: Mandala; 1990.

36. Odent M. La cientificación del amor. Madrid: Editorial Creavida; 2001.

37. Odent M. El nacimiento en la era de plástico. Tenerife: Ob Stare; 2011.

38. Kitzinger S. Birth at home. Oxford: Oxford University Press; 1979.

39. Kitzinger S. Rediscovering Birth. London: Printer \& Martin Ltd; 2000.

40. Kitzinger S. The new experience of childbirth. London: Orion Books; 2004.

41. Kitzinger S. Birth Crisis. New York: Routledge; 2006.

42. Hernández JM, Echevarría P, Gomariz MJ. La catarsis de los cuerpos grávidos. Experiencias de dolor y sufrimiento en el habitus del parto medicalizado. Revista de Dialectología y Tradiciones Populares 2016; 71(2): 487-507.

43. Organización Mundial de la Salud. Declaración de Fortaleza. Tecnología apropiada para el parto. Ginebra: OMS; 1885.

44. Organización Mundial de la Salud. Cuidados en el parto normal: una guía práctica. Ginebra: OMS; 1996.

45. Ministerio de Sanidad y Consumo. Estrategia para la atención al parto normal. Madrid: MSC; 2007.

46. Ministerio de Sanidad y Política Social. Guía de Práctica Clínica sobre la atención al parto normal. Madrid: MSPS; 2010.

47. Ministerio de Sanidad y Política Social. Estrategia Nacional de Salud Sexual y Reproductiva. Madrid: MSPS; 2010.

48. Sociedad Española de Obstetricia y Ginecología. Recomendaciones sobre la asistencia al parto normal. Madrid: SEGO; 2008.

49. Federación de Asociaciones de Matronas de España. Documento de iniciativa a favor del parto normal. Madrid: FAME; 2008.

50. Llano A. Interacciones de la biología y la antropología. En VV AA Deontología biológica. Pamplona: Editorial Eunsa; 1987: 153-169.

51. Marcel G. La dignité humaine et ses assises existentielles. Paris: Aubier-Montaigne; 1964.

52. Saraceni G. El cuerpo del delito. Reflexiones jurídico-filosóficas sobre el posthumanismo. En Ballesteros, J, Fernández, E. (Coords.) Biotecnología y Posthumanismo. Navarra: Editorial Aranzadi; 2007: 139-152.

53. Viola F. La defensa de la persona humana en la era tecnológica. En Ballesteros, J, Fernández, E. (Coords.) Biotecnología y Posthumanismo. Navarra: Editorial Aranzadi; 2007: 47-62.

54. Ferrer U. Posthumanismo y dignidad de la especie humana. En Ballesteros, J, Fernández, E. (Coords.) Biotecnología y Posthumanismo. Navarra: Editorial Aranzadi; 2007: 153-170.

55. Bacon F. La nueva Atlántida. Madrid: Editorial Aguilar; 1960[1624].

56. Caronia A. Il cyborg. Saggio sull'uomo artificiale. Milán: Editorial Shake; 2001.

57. Punzi A. Lórdine giuridico delle machina. Turín: Giappichelli editore; 2003.

58. Scheper-Hughes N, Lock MM. The mindful body: a prolegomenon to future work in Medical Anthropology. Medical Anthropology Quarterl 1987; 1(1): 6-41.

59. Menéndez EL. Hacia una práctica médica alternativa. Hegemonía y autoatención (gestión) en salud. México: Cuadernos de la Casa Chata, 86; 1984.

60. Foucault M. El nacimiento de la clinica, una arqueología de la mirada médica. Madrid: Siglo XXI; 2007 [1963].

61. Nightingale F. Notas sobre enfermería. Qué es y qué no es. Barcelona: Ed. Salvat; 1990[1859].

62. Rousseau JJ. Discurso sobre la Desigualdad entre los Hombres. London: Create Space Independent Publishing Platform; $2016[1755]$.

63. Tong R. Feminist Thought: A More Comprehensive Introduction ( $3^{\text {a }}$ edición). Colorado: Westview Press (Perseus Books); 2009.

64. Puleo A. Del ecofeminismo clásico al deconstructivo: principales corrientes de un pensamiento poco conocido. En Amorós C, De Miguel A, (eds.) Teoría feminista. De la Ilustración a la globalización. Madrid: Minerva; 2005:121-152.

65. Kant I. Fundamentación de la metafisica de las costumbres. París: Real Sociedad Económica Matritense de amigos de París, 64; 1992[1785].

66. Marcel G. Éter et avoir. Paris: Aubier-Montaigne; 1935.

67. Habermans J. El futuro de la naturaleza humana. ¿Hacia una engenesis liberal? Barcelona: Paidós; 2002. 
Culturas cosmopolitas del parto - José Manuel Hernández Garre, Baldomero de Maya Sánchez

68. Leininger M. Transcultural nursing: concepts, theories, research and practices. New York: McGraw-Hill; 2002 [1978].

69. Ehrenreich B, English D. For Her Own Good: Two Centuries of the Experts'Advice to Women. New York: Anchor Books; 1978.

70. Wagner M. Born in the USA: How a broken maternity system must be fixed to put women and children first. Berkeley: University of California Press; 2006.

71. Illich I. Némesis médica. La expropiación de la salud. Barcelona: Barral Ediciones; 1975.

72. Hernández JM, Echevarría P, Gomariz MJ. ¿Némesis obstétrica o disminución del riesgo? A debate bioético el abordaje intervencionista en los partos de bajo riesgo. Acta Bioethica 2017; 23(1): 161-170.

Recibido: 20 de diciembre de 2018

Aceptado: 26 de marzo de 2019 